\title{
Remarks on homotopy equivalence of configuration spaces of a polyhedron
}

\section{Leonid Plachta ${ }^{1}$}

Received: 4 November 2017 / Revised: 24 August 2018 / Accepted: 4 December 2018 /

Published online: 19 December 2018

(c) The Author(s) 2018

\begin{abstract}
We show that the configuration space $F_{n}(M)$ of $n$ particles in a compact connected PL manifold $M$ with nonempty boundary $\partial M$ is homotopy equivalent to the configuration space $F_{n}$ (Int $M$ ) where Int $M=M \backslash \partial M$. Actually we prove some generalization of this result for polyhedra. Similar results recently have been obtained independently for topological manifolds by Zapata (Collision-free motion planning on manifolds with boundary, 2017. arXiv:1710.00293), using different techniques. We also address the question of whether a compact PL manifold $M$ can be approximated up to homotopy type by discrete configuration spaces defined combinatorially via a simplicial subdivision of $M$.
\end{abstract}

Keywords Configuration space $\cdot$ PL manifold $\cdot$ Deformation retraction $\cdot$ Equivariant map · Polyhedron $\cdot$ Collar $\cdot$ Discrete configuration space

\section{Mathematics Subject Classification 57Q91}

\section{Introduction}

Let $X$ be a topological space and $X^{k}$ its $k$-fold Cartesian product, $k \geqslant 2$. Define the diagonal $D$ of $X^{k}$ as follows: $D=\left\{\left(x_{1}, \ldots, x_{k}\right) \in X^{k}: x_{i}=x_{j}\right.$ for some $\left.i \neq j\right\}$.

For a given topological space $X$, denote by $F_{k}(X)$ the space $X^{k} \backslash D$, the configuration space of $k$ particles in $X$ without collisions. The symmetric group $\Sigma_{k}$ acts freely on $F_{k}(X)$ by permuting coordinates of $X^{k}$. The topology of classical configuration spaces $F_{k}\left(\mathbf{R}^{n}\right)$ was studied by many authors (see, for example, [5,8] for the background). A fundamental work on this topic is the monograph by Fadell and Husseini

The research partially supported by the Polish Ministry of Science and Higher Education.

$\bowtie \quad$ Leonid Plachta

lplachta@agh.edu.pl

1 Faculty of Applied Mathematics, AGH University of Science and Technology, Al. Mickiewicza 30, 30-059 Cracow, Poland 
[7], in which the case of sphere $X=S^{m}$ is also treated. The homology structure of $F_{k}\left(\mathbf{R}^{n}\right)$ was described, for example, in [4]. It is also known that configuration spaces are not homotopy invariant even for closed manifolds (see [11]).

In this paper, we prove that if $(Q, P)$ is a pair of compact polyhedra, the subpolyhedron $P$ has a collar in $Q$, and the homotopy equivalence of the space $Q \backslash P$ and the polyhedron $Q^{\prime}$ is given by a deformation retraction of one onto another inside a collar of the subpolyhedron $P$, then it extends to a retraction of corresponding configuration spaces. It follows that if $M$ is a compact piecewise linear (PL) manifold and the homotopy equivalence of manifolds $M \backslash \partial M$ and $M^{\prime}$, where $M^{\prime} \subset M, M^{\prime} \cong M$, is given by a deformation retraction of the first one onto the other one inside a collar of the boundary $\partial M$, then it descends to a deformation of corresponding configuration spaces.

\section{Configuration spaces of polyhedra and compact manifolds with boundary}

Let $(Q, P)$ be a pair of polyhedra such that $P$ is a compact subpolyhedron of $Q$ that has a PL collar in $Q$. In this section, we compare the configuration space of the polyhedron $Q$ with the configuration space of the "open" subspace $Q \backslash P$. In particular, we will show that if $M$ is a compact PL manifold with nonempty boundary $\partial M$, then the configuration spaces $F_{k}(\operatorname{Int} M)$ and $F_{k}(M)$ are $\Sigma_{k}$-equivariantly homotopy equivalent.

Before proving a general result, we first demonstrate how our approach works in the particular case, when $M$ is a closed unit disk of the Euclidean space. Let $D^{n}$ be a closed $n$-dimensional disc in $\mathbf{R}^{n}$. The proof of the following lemma uses the techniques developed by Crowley and Skopenkov in [6].

Lemma 2.1 For each positive integer $k$ the space $F_{k}\left(D^{n}\right)$ is a deformation retract of the space $F_{k}\left(\mathbf{R}^{n}\right)$. Moreover, there is a $\Sigma_{k}$-equivariant deformation retraction of $F_{k}\left(\mathbf{R}^{n}\right)$ onto $F_{k}\left(D^{n}\right)$.

Proof Let $S^{n}$ be the $n$-dimensional sphere, $S^{n}=\mathbf{R}^{n} \cup\{\infty\}$. Decompose $S^{n}$ into two half-spheres, $S_{0}$ and $S_{\infty}$, where $S_{0}=\left\{w \in \mathbf{R}^{n}:|w| \leqslant 1\right\}$ and $S_{\infty}=\operatorname{cl}\left(S^{n} \backslash S_{0}\right)$. Consider the subspace $R=S^{n} \backslash\{0\}$ of $S^{n}$ which is obviously homeomorphic to $\mathbf{R}^{n}$. There is a $\Sigma_{k}$-equivariant deformation retraction $g_{t}$ of $F_{k}(R)$ on $F_{k}\left(S_{\infty}\right)$. To show this, consider in $\mathbf{R}^{n} \subset S^{n}$ a closed disc $D_{2}$ of radius 2 centered at 0 . The half-sphere $S_{0}$ is identified with a closed unit disc $D_{1}$.

For each $s=1, \ldots, k$, define a map $f_{s}: F_{k}(R) \rightarrow S_{\infty}$ as follows:

(i) $f_{s}\left(x_{1}, \ldots, x_{k}\right)=x_{s}$ if no $x_{i}, i=1, \ldots, k$, is contained in Int $D_{1}$;

(ii) If $x=\left(x_{1}, \ldots, x_{k}\right) \in F_{k}(R) \backslash F_{k}\left(S_{\infty}\right)$, take any $j$ such that $\min _{s}\left\{\left|x_{s}\right|\right\}=\left|x_{j}\right|$. Denote $\left|x_{j}\right|$ by $\rho$. We obviously have $0<\rho<1$. Put

$$
f_{s}\left(x_{1}, \ldots, x_{k}\right)=\frac{x_{s}}{\left|x_{s}\right|} \frac{2-2 \rho+\left|x_{s}\right|}{2-\rho} \quad \text { if } \quad x_{s} \in D_{2} ;
$$

(iii) $f_{s}\left(x_{1}, \ldots, x_{k}\right)=x_{s}$ if $x_{s}$ is not in Int $D_{2}$. 
Each coordinate function $f_{s}$ is fixed on points $x \in F_{k}\left(S_{\infty}\right)$ and on the points $x=\left(x_{1}, \ldots, x_{s}, \ldots, x_{k}\right) \in F_{k}(R) \backslash F_{k}\left(S_{\infty}\right)$ with $\left|x_{s}\right| \geqslant 2$. For the points $\left(x_{1}, \ldots, x_{s}, \ldots, x_{k}\right) \in F_{k}\left(S_{\infty}\right)$ with $\left|x_{s}\right| \leqslant 2$, it acts along the rays in $\mathbf{R}^{n}$ originating at 0 . In this case, it looks like a monotone PL function $h:[0,2] \rightarrow[0,2]$ with $h(0)=0, h(2)=2$ and $h(\rho)=1$, which is linear on the intervals $[0, \rho]$ and $[\rho, 2]$. In particular, if $\left|x_{s}\right|=\rho$, where $x_{s}$ is the $s$-th coordinate of the point $\left(x_{1}, \ldots, x_{k}\right)$, we have $\left|f_{s}(x)\right|=1$. Substituting $\left|x_{s}\right|=2$ in the formula $\frac{x_{s}}{\left|x_{s}\right|} \frac{2-2 \rho+\left|x_{s}\right|}{2-\rho}$, we get $f_{s}(x)=x_{s}$. Moreover if $\rho<\left|x_{s}\right|<2$, then $1<\left|f_{s}\left(x_{1}, \ldots, x_{k}\right)\right|<2$. It follows that each function $f_{s}$ is well defined.

It is clear that $f_{s}$ is continuous at $x \in F_{k}\left(S_{\infty}\right)$. If $x \in F_{k}(R) \backslash F_{k}\left(S_{\infty}\right)$ and $x_{s}$ is in the exterior of the disc $D_{2}$ or in $\partial D_{2}$, the function $f_{s}$ depends only on $x_{s}$ and we have $f_{s}(x)=x_{s}$. If $x \in F_{k}(R) \backslash F_{k}\left(S_{\infty}\right)$ and $x_{s}$ is in the interior of the disc $D_{2}$, the value $f_{s}(x)$ depends continuously on the parameter $\rho$. On the other hand, the function $\rho$ is the minimum of finite number of continuous functions (the norms $\left|x_{i}\right|$ ). So within a small neighborhood $U(x)$ the parameter $\rho(x)$ also changes very little. It follows that $f_{s}$ is continuous at the points $x \in F_{k}(R) \backslash F_{k}\left(S_{\infty}\right)$ with $\left|x_{s}\right|<2$. Finally if $x \in F_{k}(R) \backslash F_{k}\left(S_{\infty}\right)$ and $\left|x_{s}\right|=1$, the above remarks and formula (ii) show that $f_{s}$ is continuous also at the point $x$.

Define a map $f: F_{k}(R) \rightarrow\left(S_{\infty}\right)^{k}$ by the formula $f=\left(f_{1}, \ldots, f_{s}, \ldots, f_{k}\right)$. It follows that $f_{s}$ is the $s$-th coordinate function of $f$ and the map $f$ itself is continuous. Actually $f$ maps the points $x=\left(x_{1}, \ldots, x_{s}, \ldots, x_{k}\right)$ with distinct coordinates $x_{s}$ to the points $y=\left(y_{1}, \ldots, y_{s}, \ldots, y_{k}\right)$ with different coordinates $y_{s}$. This is obvious for the points $x \in F_{k}\left(S_{\infty}\right)$ and for the points $x=\left(x_{1}, \ldots, x_{s}, \ldots, x_{k}\right)$ such that all $x_{s}$ lie on different rays of the space $\mathbf{R}^{n}$. On the other hand, if some coordinates $x_{i}$ and $x_{j}$ of $x \in F_{k}(R) \backslash F_{k}\left(S_{\infty}\right)$ are on the same ray, then $\left|x_{i}\right| \neq\left|x_{j}\right|$ and $\left|f_{i}(x)\right| \neq\left|f_{j}(x)\right|$, according to the monotonic property of each coordinate function $f_{s}$. Therefore $f$ maps the configuration space $F_{k}(R)$ onto the configuration space $F_{k}\left(S_{\infty}\right)$. By the properties of the coordinate functions $f_{s}, f$ retracts the space $F_{k}(R)$ onto the space $F_{k}\left(S_{\infty}\right)$. Moreover it is not difficult to see that $f$ is actually a $\Sigma_{k}$-equivariant retraction of $F_{k}(R)$ onto $F_{k}\left(S_{\infty}\right)$.

Each map $f_{s}$ obviously admits an extension to a homotopy $g_{t}^{s}$ via the following formula:

$$
g_{t}^{s}\left(x_{1}, \ldots, x_{s}, \ldots, x_{k}\right)=(1-t) x_{s}+t f_{s}\left(x_{1}, \ldots, x_{s}, \ldots, x_{k}\right), \quad 0 \leqslant t \leqslant 1 .
$$

For each $s, 1 \leqslant s \leqslant k$, and each $x \in F_{k}\left(S_{\infty}\right)$ the homotopy $g_{t}^{s}$ keeps the coordinate $x_{s}$ of $x$ point-wise fixed. Put $g_{t}=\left(g_{t}^{1}, \ldots, g_{t}^{k}\right)$ for each $t \in[0,1] . g_{t}$ is obviously a $\Sigma_{k}$-equivariant deformation retraction of $F_{k}(R)$ onto $F_{k}\left(S_{\infty}\right)$.

Let $Q$ be a polyhedron and $P$ its compact subpolyhedron which has a collar in $Q$. A closed collar of $P$ in $Q$ is represented by the image of PL embedding $h: P \times[0,2] \rightarrow$ $Q$ where $h(P \times\{0\})$ is identified with $P$. It is a regular neighborhood of $P$ in $Q$ [10]. Denote by $U$ a small open collar of $P$ in $Q$ which is identified with the image $h(P \times[0,1))$. Obviously, $Q \backslash U$ is homeomorphic to $Q$. It follows that $F_{k}(Q \backslash U)$ and $F_{k}(Q)$ are homeomorphic in a natural way. 
Theorem 2.2 For each $k$ the space $F_{k}(Q \backslash P)$ deformation retracts onto the subspace $F_{k}(Q \backslash U)$. Moreover, the configuration space $F_{k}(Q)$ is $\Sigma_{k}$-equivariantly homotopy equivalent to the configuration space $F_{k}(Q \backslash P)$.

Proof Let $R_{1}, \ldots, R_{m}$ be the connected components of $P$. Moreover, let $C_{i}, i=$ $1, \ldots, m$, be the closed collars of $R_{1}, \ldots, R_{m}$, respectively, in $Q$ where each $C_{i}$ is identified with $R_{i} \times[0,2], i=1, \ldots, m$, via the PL embedding $h$ and $R_{i}$ is identified with $R_{i} \times\{0\}$ and $C_{i} \cap C_{j}=\varnothing$ if $i \neq j$. We also identify $U=\bigcup_{i=1}^{m}\left(R_{i} \times[0,1)\right)$ with an open collar of $P$ in $Q$ as before. Put $C=\bigcup_{i=1}^{m} C_{i}$.

By the above identification, each $z \in C$ can be uniquely represented as $z=(x, \tau)$ where $x \in R_{j}$ for some $j$ and $0 \leqslant \tau \leqslant 2$. Now we define a deformation retraction of the space $F_{k}(Q \backslash P)$ onto the space $F_{k}(Q \backslash U)$ as follows.

For each $\rho, 0<\rho<1$, take a monotone PL function $h_{\rho}:[0,2] \rightarrow[0,2]$ such that $h_{\rho}(0)=0, h_{\rho}(2)=2$ and $h_{\rho}(\rho)=1$. For $\rho \leqslant \lambda \leqslant 2$, the function $h_{\rho}$ can be expressed as follows: $h(\lambda)=\frac{2-2 \rho+\lambda}{2-\rho}$ (see also the proof of Lemma 2.1). First, for each $1 \leqslant s \leqslant k$ we shall define a map $f_{s}: F_{k}(Q \backslash P) \rightarrow Q \backslash U$. Let $y=\left(y_{1}, \ldots, y_{k}\right) \in F_{k}(Q \backslash P)$. Let $V=\left\{y_{i_{1}}, \ldots, y_{i_{r}}\right\}$ be the set of coordinates of $y$ which belong to the open collar $U$ of $P$. If $V=\varnothing$, we put $f_{s}(y)=y$. Assume that $V \neq \varnothing$. We have $y_{i_{s}}=\left(x_{i_{s}}, \tau_{s}\right)$ for each $1 \leqslant s \leqslant r$ where $x_{i_{s}} \in P$. Take any $l$ such that $\min _{s}\left\{\tau_{s}\right\}=\tau_{l}$ where $s$ runs from 1 to $r$. Denote $\tau_{l}$ by $\rho$. We obviously have $0<\rho<1$. Now, the expression for the coordinate function $f_{s}$ is the following:

- $f_{s}\left(y_{1}, \ldots, y_{k}\right)=y_{s}$ if no $y_{i}$ belongs to $U, i=1, \ldots, k$;

- $f_{s}\left(y_{1}, \ldots, y_{k}\right)=y_{s}$ if some $y_{i}$ belongs to $U, i=1, \ldots, k$, but $y_{s}$ does not belong to $C \backslash P$;

- $f_{s}\left(y_{1}, \ldots y_{k}\right)=\left(x_{s}, h_{\rho}\left(\tau_{s}\right)\right)$, if $\left(y_{1}, \ldots, y_{k}\right) \in F_{k}(Q \backslash P) \backslash F_{k}(Q \backslash U)$ and $y_{s} \in$ $C \backslash P$, where $y_{s}=\left(x_{s}, \tau_{s}\right), x_{s} \in P, 0<\tau_{s} \leqslant 2$.

Note that if the coordinate $y_{s}$ of $y=\left(y_{1}, \ldots, y_{k}\right)$ belongs to the collar $C$, i.e., $y_{s}=\left(x_{s}, \tau_{s}\right)$ where $x_{s} \in P$ and $\rho(y) \leqslant \tau_{s} \leqslant 2$, then the $s$-th coordinate of $f_{s}\left(y_{1}, \ldots, y_{s}, \ldots, y_{k}\right)$ can be represented as $\left(x_{s}, \lambda_{s}\right)$ where $1 \leqslant \lambda_{s} \leqslant 2$. Moreover if $\tau_{s}=2$, then $\lambda_{s}=2$ and if $\tau_{s}=\rho$, then $\lambda_{s}=1$.

It follows that for each $s=1, \ldots, k$ the map $f_{s}: F_{k}(Q \backslash P) \rightarrow Q \backslash U$ is well defined in its domain. The continuity of $f_{s}$ is performed along the same line as the one of the coordinate functions in the proof of Lemma 2.1. We omit the details.

Therefore the map $f=\left(f_{1}, \ldots, f_{s}, \ldots, f_{k}\right): F_{k}(Q \backslash P) \rightarrow(Q \backslash U)^{k}$ is also continuous. Let $y=\left(y_{1}, \ldots, y_{k}\right)$ be any point of the configuration space $F_{k}(Q \backslash P)$ and let $f_{i}$ and $f_{j}$ be two coordinate functions of the map $f$ where $i \neq j$. If $y \in$ $F_{k}(Q \backslash U)$, we have $f_{i}(y)=y_{i} \neq y_{j}=f_{j}(y)$. Now assume that some coordinate $y_{s}$ of $y$ is in the set $U$. If one of the coordinates $y_{i}$ and $y_{j}$ is outside the collar $C$, it follows immediately that $f_{i}(y) \neq f_{j}(y)$. Assume that both $y_{i}$ and $y_{j}$ belong to the collar $C$. The coordinates $y_{i}$ and $y_{j}$ have the following presentation: $y_{i}=\left(x_{i}, \tau_{i}\right)$ and $y_{j}=\left(x_{j}, \tau_{j}\right)$ where $x_{i}, x_{j} \in P$ and $\rho(y) \leqslant \tau_{i}, \tau_{j} \leqslant 2$. If $x_{i} \neq x_{j}$ it follows immediately that $f_{i}(y) \neq f_{j}(y)$. On the other hand, if $x_{i}=x_{j}$, then $\tau_{i} \neq \tau_{j}$. By the monotonic property of the function $h_{\rho}$, we get $h_{\rho}\left(\tau_{i}\right) \neq h_{\rho}\left(\tau_{j}\right)$ which implies that $f_{i}(y) \neq f_{j}(y)$. It follows that $f$ maps $k$-tuples $\left(y_{1}, \ldots, y_{k}\right)$ with distinct coordinates into $k$-tuples $\left(z_{1}, \ldots, z_{k}\right)$ with distinct coordinates. Therefore $f$ is actually a map 
from the configuration space $F_{k}(Q \backslash P)$ onto the configuration $F_{k}(Q \backslash U)$. Moreover, by the properties of the coordinate functions $f_{s}, f$ is a $\Sigma_{k}$-equivariant retraction of the space $F_{k}(Q \backslash P)$ onto the subspace $F_{k}(Q \backslash U)$.

The map $f$ can be extended to the deformation retraction $g_{t}^{\prime}: F_{k}(Q \backslash P) \rightarrow$ $F_{k}(Q \backslash P), t \in[0,1]$, with $g_{0}^{\prime}=\operatorname{id}_{F_{k}(Q \backslash P)}$ and $g_{1}^{\prime}=f$. The deformation retraction $g_{t}^{\prime}$ is defined in the same way as the homotopy $g_{t}$ in the proof of Lemma 2.1. We omit the details. Since the map $f$ is $\Sigma_{k}$-equivariant, we can arrange that the deformation retraction $g_{t}^{\prime}$ of the space $F_{k}(Q \backslash P)$ onto the space $F_{k}(Q \backslash U)$ is also $\Sigma_{k}$-equivariant. This completes the proof of the theorem.

Let $M$ be a connected, compact and smooth or PL manifold with the nonempty boundary $\partial M$. Then $\partial M$ is collared in $M$. Moreover we have the following

Corollary 2.3 For each $k \geqslant 1$ the configuration space $F_{k}(M)$ is $\Sigma_{k}$-equivariantly homotopy equivalent to the configuration space $F_{k}(\operatorname{Int} M)$.

\section{Discrete configuration spaces of complexes}

Let $K$ be a finite simplicial complex. Denote by $|K|$ the underlying topological space of $K$ which is a polyhedron. For each $k \leqslant n$ the subcomplex $D_{n}(K)$ of the cell complex $K^{n}$ is defined in the following way: $D_{n}(K)=\bigcup \sigma_{1} \times \cdots \times \sigma_{n}$ where the sum is over all $n$ pairwise disjoint closed cells in $K$ (see [2,3]). The subcomplex $D_{n}(K)$ is called the discrete configuration space of the complex $K$ with the parameter $n$. This is the largest cell complex that is contained in the product $K^{n}$ minus its diagonal $\left\{\left(x_{1}, \ldots, x_{n}\right) \in|K|^{n}: x_{i}=x_{j}\right.$ for some $\left.i \neq j\right\}$. The symmetric group $\Sigma_{n}$ acts naturally on $D_{n}(K)$ by permuting the cells in the product. The polyhedron $\left|D_{n}(K)\right|$ has natural $\Sigma_{n}$-equivariant embedding in the configuration space $F_{n}(|K|)$ for each $n \geqslant 2$.

A graph $G$ can be considered as a 1-complex. Abrams [1] proved that for each graph $G$ there is a subdivision $G^{\prime}$ of $G$ such that the discrete configuration space $D_{n}\left(G^{\prime}\right)$ is homotopy equivalent to the usual configuration space $F_{n}(G), n \geqslant 2$.

The problem of a cell approximation of the space $F_{n}(X)$ where $X$ is a polyhedron of dimension $\geqslant 2$ was considered and studied in [3]. For $n=2$, Hu [9] showed that the configuration spaces $D_{2}(K)$ and $F_{2}(K)$ are homotopy equivalent. Moreover he showed that for any finite simplicial complex $K$ there is a $\Sigma_{2}$-equivariant deformation retraction of $F_{2}(K)$ onto $\left|D_{2}(K)\right|$. In general, the problem can be formulated as follows

Problem Let $X$ be a compact connected PL manifold of dimension $k \geqslant 2$ and let $n>2$. Show that there is a subdivision $K$ of $X$ such that the manifold $F_{n}(X)$ admits a $\Sigma_{n}$-equivariant deformation retraction onto the polyhedron $\left|D_{n}(K)\right|$ or give a counterexample.

To the best of our knowledge, for PL manifolds of dimension $k \geqslant 2$, the question of cell approximation of configuration spaces remains open.

Acknowledgements The author thanks the referee for indicating some mistakes and inaccuracies in definitions in the earlier version of the paper, and for useful remarks and valuable comments. 
Open Access This article is distributed under the terms of the Creative Commons Attribution 4.0 International License (http://creativecommons.org/licenses/by/4.0/), which permits unrestricted use, distribution, and reproduction in any medium, provided you give appropriate credit to the original author(s) and the source, provide a link to the Creative Commons license, and indicate if changes were made.

\section{References}

1. Abrams, A.D.: Configuration Spaces of Braid Groups of Graphs. Ph.D. thesis, University of California, Berkeley (2000)

2. Abrams, A., Gay, D., Hower, V.: Discretized configurations and partial partitions. Proc. Amer. Math. Soc. 141(3), 1093-1104 (2013)

3. An, B.H., Drummond-Cole, G.C., Knudsen, B.: Subdivisional spaces and graph braid groups (2017). arXiv:1708.02351v1

4. Bödigheimer, C.-F., Cohen, F., Taylor, L.: On the homology of configuration spaces. Topology 28(1), 111-123 (1989)

5. Cohen, F.R.: Introduction to configuration spaces and their applications. In: Berrick, A.J., et al. (eds.) Braids. Lecture Notes Series, Institute for Mathematical Sciences, National University of Singapore, vol. 19, pp. 183-261. World Scientific, Hackensack (2009)

6. Crowley, D., Skopenkov, A.: Embeddings of non-simply-connected 4-manifolds in 7-space III. Piecewise-linear classification (preprint)

7. Fadell, E.R., Husseini, S.Y.: Geometry and Topology of Configuration Spaces. Springer Monographs in Mathematics. Springer, Berlin (2001)

8. Fadell, E., Neuwirth, L.: Configuration spaces. Math. Scand. 10, 111-118 (1962)

9. Hu, S.: Isotopy invariants of topological spaces. Proc. Roy. Soc. London Ser. A 255, 331-366 (1960)

10. Hudson, J.F.P.: Piecewise Linear Topology. University of Chicago Lecture Notes prepared with the assistance of J. L. Shaneson and J. Lees. W.A. Benjamin, New York (1969)

11. Longoni, R., Salvatore, P.: Configuration spaces are not homotopy invariant. Topology 44(2), 375-380 (2005)

12. Zapata, C.A.I.: Collision-free motion planning on manifolds with boundary (2017). arXiv: 1710.00293

Publisher's Note Springer Nature remains neutral with regard to jurisdictional claims in published maps and institutional affiliations. 